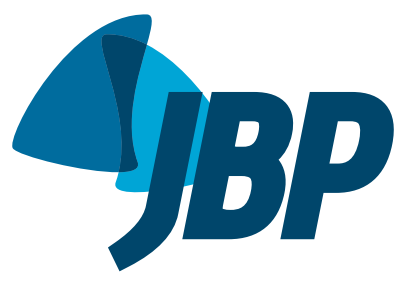

\title{
Translation and cultural adaptation of the European Organisation for Research and Treatment of Cancer Quality of Life Questionnaire-Lung Cancer Module for quality of life assessment in patients with lung cancer in Brazil
}

Ana Paula Ramos Marinho, 1,2,a, Gracielle Fin ${ }^{3,4, b}$, Antuani Rafael Baptistella ${ }^{3, c}$, Rudy José Nodari Júnior ${ }^{3, d}$, Magnus Benetti ${ }^{4, e}$

1. Departamento de Fisioterapia, Centro Universitário Unifacvest Lages (SC) Brasil.

2. Departamento de Fisioterapia Universidade do Estado de Santa Catarina -UDESC/CEFID Florianópolis (SC) Brasil.

3. Área das Ciências da Vida, Universidade do Oeste de Santa Catarina - Unoesc Joaçaba (SC) Brasil.

4. Departamento de Educação Física, Universidade do Estado de Santa Catarina -UDESC/CEFID Florianópolis (SC) Brasil.

a. (D) http://orcid.org/0000-0001-7469-1092

b. (D) http://orcid.org/0000-0001-7860-4451

c. (iD http://orcid.org/0000-0003-1708-9921

d. (D) http://orcid.org/0000-0002-8375-657X

e. (D) http://orcid.org/0000-0002-0079-255X

Submitted: 10 January 2018 Accepted: 7 December 2018

Study carried out in the Setor de Oncologia, Hospital Universitário Santa Terezinha, Joaçaba (SC) Brasil.

\begin{abstract}
Objective: To translate the European Organisation for Research and Treatment of Cancer (EORTC) 29-item Quality of Life Questionnaire-Lung Cancer Module (QLQLC29, developed for the assessment of quality of life in patients with lung cancer) to Portuguese, conducting a pilot study of the Portuguese-language version and adapting it for use in Brazil. Methods: For the translation, cultural adaptation, and pilot testing of the QLQ-LC29, we followed the guidelines established by the EORTC. The translation (English $\rightarrow$ Portuguese) and back-translation (Portuguese $\rightarrow$ English) were both carried out by translators, working independently, who were native speakers of one language and fluent in the other. After review, a draft version was created for pilot testing in lung cancer patients in Brazil. Results: A total of 15 patients diagnosed with lung cancer completed the Portuguese-language version of the questionnaire. At the end of the process, we conducted a structured interview to identify any patient difficulty in understanding any of the questions. The final versions were sent to the EORTC and were approved. Conclusions: The Portuguese-language version of the EORTC QLQ-LC29 appears to be a useful, important, reliable questionnaire that is a valid tool for assessing quality of life in patients with lung cancer in Brazil.
\end{abstract}

Keywords: Surveys and Questionnaires; Lung neoplasms; Quality of Life; Brazil; Translations.

\section{INTRODUCTION}

Over the past 100 years, lung cancer has changed from a rare disease to a real global problem. Scientific and clinical efforts to improve outcomes have led to a better understanding not only of the etiology of lung cancer but also of the histological and molecular characteristics of individual lung tumors. ${ }^{(1)}$

Lung cancer is the most commonly diagnosed cancer (11.6\% of all cancer cases) and the leading cause of cancer death ( $18.4 \%$ of all cancer deaths) worldwide; its prevalence is increasing among women and has surpassed that of breast cancer in 28 countries. ${ }^{(2)}$ In Brazil, lung cancer is considered the most deadly type of cancer among men and women. A total of 18,740 and 12,530 new cases of lung cancer are estimated among men and women, respectively, for each year of the 2018-2019 biennium. This corresponds to an estimated risk of 18.16 new cases per 100,000 men and an estimated risk of 11.81 new cases per 100,000 women, lung cancer being the second most common type of cancer in Brazil. ${ }^{(3)}$
Although lung cancer is predominantly caused by tobacco smoke, approximately $25 \%$ of all lung cancers worldwide are not attributable to this etiology. ${ }^{(4)}$ Other etiologies include environmental exposure to smoke, radiation, or smoke from burning wood; occupational exposures; oncogenic viruses; genetic alterations; and changes in sex hormone levels. ${ }^{(4,5)}$

The concept of quality of life (QoL) is broad, subjective, and encompasses four main domains: physical wellbeing; psychological well-being; social well-being; and occupational well-being. ${ }^{(6,7)}$ In cancer patients, QoL care and attention to QoL are even greater, because QoL can not only be a predictor of morbidity and mortality but can also serve as a parameter for evaluating treatment course and response.

Several generic QoL scales are used in Brazil and worldwide; however, it is important that the instrument chosen for use be as specific as possible so that it can provide information as accurately as possible. With this in mind, the European Organisation for Research and 
Treatment of Cancer (EORTC) developed a variety of instruments to assess QoL in patients with cancer. ${ }^{\left({ }^{(8)}\right.}$ The core questionnaire is the EORTC Quality of Life Questionnaire Core 30 (QLQ-C30), which includes five functional scales, three symptom scales, and a global health and QoL scale. ${ }^{(9)}$ However, the use of the EORTC QLQ-C30 prompted the need for instruments that could assess the specificity and particularities of each type of cancer more specifically, which resulted in a multitude of modules for different types of cancer. ${ }^{(10)}$ One of these modules is known as the 29-item QLQ-Lung Cancer Module (QLQ-LC29), the objective of which is to assess QoL in patients with lung cancer. ${ }^{(11,12)}$ The EORTC QLQ-LC29 was developed as an update to the previous lung cancer module, the EORTC QLQ-LC13, because of important advances in lung cancer treatment and in QoL assessment. ${ }^{(11)}$

The objective of the present study was to translate the original English-language version of the EORTC QLQ-LC29 to Portuguese, adapting it for use in Brazil, in order to provide an appropriate tool for assessing QoL in patients with lung cancer in the country.

\section{METHODS}

This was a methodological study whose objective was to translate and culturally adapt the QLQ-LC29, which is a module for specifically assessing QoL in patients with lung cancer. The final version has 29 items and refers to a specific time period (i.e., "during the past week"). Patients also have the opportunity to report whether there were symptoms or problems that were not covered by the questionnaire, but were relevant for them during the past week. Each item is scored on a 4-point scale (Not at All; A Little; Quite a Bit; and Very Much).

\section{Translation procedure}

The translation was made along with the cultural adaptation and the pilot testing authorized by the EORTC Quality of Life Department and carried out in accordance with the EORTC translation procedure. ${ }^{(13)}$

The original English-language version was translated by two translators, working independently, who were native speakers of Portuguese and fluent in English. Subsequently, a reconciled translation was made on the basis of the two translations, that is, a third person analyzed the two translations to achieve the best possible version by choosing one of the two translations or by combining them on the basis of their similarities, wording, etc. The next step was to translate the reconciled version back into English, which was done by two translators who were native speakers of or fluent in English. The result of these steps (forward translation, reconciliation, and backward translation with comments) was put into a translation report that was reviewed by the EORTC translation unit. In the review of the report, all suggestions and corrections were analyzed and discussed. Once the discussion reached a consensus, the translation could undergo linguistic validation (pilot testing).

\section{Pilot testing}

Pilot testing, in accordance with the EORTC translation procedure, ${ }^{(13)}$ includes a group of 10 to 15 patients, who are invited to complete the questionnaire. After completion of the questionnaire, a structured interview focusing on each item, one by one, is conducted to investigate whether participants would report any difficulty answering the questions and whether they found any item to be confusing, upsetting, or offensive, or to contain difficult vocabulary. All of the participants' comments should be pooled and summarized in a pilot-testing report, which should be sent for review to the EORTC translation unit. Once all comments have been analyzed and discussed, the EORTC translation unit prepares the final version of the translation and closes the project.

\section{Participants}

This was a convenience sample in which patients were randomly recruited from the Oncology Department of the Santa Terezinha University Hospital, located in the city of Joaçaba, Brazil. Patient status was determined from the medical records, and only patients with a diagnosis of lung cancer were considered study participants.

Inability to understand or complete the questionnaire was considered an exclusion criterion. There were no restrictions regarding gender, age, or level of education. All participating patients were receiving cancer treatment and were approached at the time of their medical visit. It should be highlighted that the EORTC procedures for questionnaire translation do not stipulate a single time point during the course of the disease for patient assessment, this time point (diagnosis, treatment, or control) being random. The study was approved by the Human Research Ethics Committee of the Universidade do Oeste de Santa Catarina and the Santa Terezinha University Hospital (Protocol no. 2.286.701 of September 20, 2017).

\section{Statistical analysis}

Once the interviews were completed, all data were compiled and analyzed using simple descriptive statistics. Understandability was assessed using a Likert scale preceded by the question, "Did you understand what was asked?"-0: I did not understand anything; 1 : I understood only a little; 2 : I somewhat understood it; 3 : I understood almost everything, but I have some questions; 4 : I understood almost everything; and 5 : I understood it perfectly well, and I have no questions. ${ }^{(14)}$ The internal consistency of the scale was calculated with Cronbach's alpha coefficient. All statistical analyses were performed with the IBM SPSS Statistics software package, version 20.0 (IBM Corporation, Armonk, NY, USA).

\section{RESULTS}

Fifteen patients participated in the present study, 8 of whom were female. The mean age was $61 \pm$ 5.2 years. Thirteen patients $(86.7 \%)$ had attended only elementary school, and 2 patients (13.3\%) had completed high school. 
Table 1. Original English-language version, translations, back-translations, and final Brazilian Portuguese-language version of the Quality of Life Questionnaire-Lung Cancer 29.

\begin{tabular}{|c|c|c|c|c|c|}
\hline$\#$ & $\begin{array}{l}\text { Original English- } \\
\text { language version }\end{array}$ & Translation & $\begin{array}{l}\text { Selected } \\
\text { translation }\end{array}$ & Back-translation & $\begin{array}{c}\text { Final Brazilian } \\
\text { Portuguese- } \\
\text { language version }\end{array}$ \\
\hline 1 & Have you coughed? & $\begin{array}{l}\text { (T1) Você tem tido tosse? } \\
\text { (T2) Você tem tossido? }\end{array}$ & $\mathrm{T} 2$ & $\begin{array}{l}\text { (R1) Have you coughed? } \\
\text { (R2) Have you coughed up? }\end{array}$ & Você teve tosse? \\
\hline 2 & $\begin{array}{l}\text { Have you coughed } \\
\text { up blood? }\end{array}$ & $\begin{array}{l}\text { (T1) Tossiu sangue? } \\
\text { (T2) Você tem tossido } \\
\text { sangue? }\end{array}$ & $\mathrm{T} 2$ & $\begin{array}{l}\text { (R1) Have you coughed up } \\
\text { blood? } \\
\text { (R2) Have you coughed up } \\
\text { blood? }\end{array}$ & $\begin{array}{l}\text { Você teve tosse } \\
\text { com sangue? }\end{array}$ \\
\hline 3 & $\begin{array}{l}\text { Have you been } \\
\text { short of breath } \\
\text { when you rested? }\end{array}$ & $\begin{array}{l}\text { (T1) Sentiu falta de ar } \\
\text { enquanto repousava? } \\
\text { (T2) Você sentiu falta de ar } \\
\text { quando descansava? }\end{array}$ & $\mathrm{T} 2$ & $\begin{array}{l}\text { (R1) Have you been } \\
\text { breathless when you rested? } \\
\text { (R2) Have you been short of } \\
\text { breath when you rested? }\end{array}$ & $\begin{array}{l}\text { Você teve falta } \\
\text { de ar quando } \\
\text { descansava? }\end{array}$ \\
\hline 4 & $\begin{array}{l}\text { Have you been } \\
\text { short of breath } \\
\text { when you walked? }\end{array}$ & $\begin{array}{l}\text { (T1) Sentiu falta de ar } \\
\text { enquanto andava? } \\
\text { (T2) Você sentiu falta de ar } \\
\text { quando caminhava? }\end{array}$ & $\mathrm{T} 2$ & $\begin{array}{l}\text { (R1) Have you been } \\
\text { breathless when you walked? } \\
\text { (R2) Have you been short of } \\
\text { breath when you rested? }\end{array}$ & $\begin{array}{l}\text { Você teve falta } \\
\text { de ar quando } \\
\text { caminhava? }\end{array}$ \\
\hline 5 & $\begin{array}{l}\text { Have you been } \\
\text { short of breath } \\
\text { when you climbed } \\
\text { stairs? }\end{array}$ & $\begin{array}{l}\text { (T1) Sentiu falta de ar } \\
\text { enquanto subia escadas (se } \\
\text { subisse)? } \\
\text { (T2) Você sentiu falta de } \\
\text { ar enquanto subia escadas } \\
\text { quando subiu escadas? (se } \\
\text { subiu) }\end{array}$ & $\mathrm{T} 2$ & $\begin{array}{l}\text { (R1) Have you been } \\
\text { breathless when you climbed } \\
\text { stairs? } \\
\text { (R2) Have you been short of } \\
\text { breath when you climbed } \\
\text { stairs? }\end{array}$ & $\begin{array}{l}\text { Você teve falta de } \\
\text { ar quando subiu } \\
\text { escadas? }\end{array}$ \\
\hline 6 & $\begin{array}{l}\text { Have you had a } \\
\text { sore mouth or } \\
\text { tongue? }\end{array}$ & $\begin{array}{l}\text { (T1) Sentiu sua boca ou } \\
\text { língua doloridas? } \\
\text { (T2) Você sentiu dor na boca } \\
\text { ou língua? }\end{array}$ & $\mathrm{T} 2$ & $\begin{array}{l}\text { (R1) Have you had a sore } \\
\text { mouth or tongue? } \\
\text { (R2) Have you had a sore } \\
\text { mouth or tongue? }\end{array}$ & $\begin{array}{l}\text { Você teve sua } \\
\text { boca ou língua } \\
\text { doloridas? }\end{array}$ \\
\hline 7 & $\begin{array}{l}\text { Have you had } \\
\text { problems } \\
\text { swallowing? }\end{array}$ & $\begin{array}{l}\text { (T1) Sentiu dificuldade ao } \\
\text { engolir? } \\
\text { (T2) Você sentiu dificuldade } \\
\text { para engolir? }\end{array}$ & $\mathrm{T} 2$ & $\begin{array}{l}\text { (R1) Have you had problems } \\
\text { swallowing? } \\
\text { (R2) Have you had problems } \\
\text { swallowing? }\end{array}$ & $\begin{array}{l}\text { Você teve } \\
\text { problemas para } \\
\text { engolir? }\end{array}$ \\
\hline 8 & $\begin{array}{l}\text { Have you had } \\
\text { tingling hands or } \\
\text { feet? }\end{array}$ & $\begin{array}{l}\text { (T1) Você teve sensação de } \\
\text { formigamento nas mãos ou } \\
\text { pés? } \\
\text { (T2) Teve dormência } \\
\text { (formigamento) nas mãos } \\
\text { ou pés? }\end{array}$ & $\mathrm{T} 1$ & $\begin{array}{l}\text { (R1) Have you had tingling } \\
\text { hands or feet? } \\
\text { (R2) Have you had numbness } \\
\text { on hands or feet? }\end{array}$ & $\begin{array}{l}\text { Você teve } \\
\text { sensação de } \\
\text { formigamento nas } \\
\text { mãos ou pés? }\end{array}$ \\
\hline 9 & $\begin{array}{l}\text { Have you had hair } \\
\text { loss? }\end{array}$ & $\begin{array}{l}\text { (T1) Você tem queda de } \\
\text { cabelo? } \\
\text { (T2)Você já perdeu cabelo? }\end{array}$ & $\mathrm{T} 1$ & $\begin{array}{l}\text { (R1) Have you had hair loss? } \\
\text { (R2) Have you had hair loss? }\end{array}$ & $\begin{array}{l}\text { Você teve queda } \\
\text { de cabelo? }\end{array}$ \\
\hline 10 & $\begin{array}{l}\text { Have you had pain } \\
\text { in your chest? }\end{array}$ & $\begin{array}{l}\text { (T1) Você tem sentido dores } \\
\text { no peito? } \\
\text { (T2) Você já sentiu dores no } \\
\text { peito? }\end{array}$ & $\mathrm{T} 1$ & $\begin{array}{l}\text { (R1) Have you had pain in } \\
\text { your chest? } \\
\text { (R2) Have you had pain in } \\
\text { your chest? }\end{array}$ & $\begin{array}{l}\text { Você teve dores } \\
\text { no peito? }\end{array}$ \\
\hline 11 & $\begin{array}{l}\text { Have you had pain } \\
\text { in your arm or } \\
\text { shoulder? }\end{array}$ & $\begin{array}{l}\text { (T1) Você sentiu dores no } \\
\text { braço ou ombro? } \\
\text { (T2) Você sentiu dores no } \\
\text { braço ou ombro? }\end{array}$ & $\mathrm{T} 1=\mathrm{T} 2$ & $\begin{array}{l}\text { (R1) Have you had pain in } \\
\text { your arm or shoulder? } \\
\text { (R2) Have you had pain in } \\
\text { your arm or shoulder? }\end{array}$ & $\begin{array}{l}\text { Você teve dores } \\
\text { no braço ou } \\
\text { ombro? }\end{array}$ \\
\hline 12 & $\begin{array}{l}\text { Have you had pain } \\
\text { in other parts of } \\
\text { your body? }\end{array}$ & $\begin{array}{l}\text { (T1) Sentiu dores em outras } \\
\text { partes de seu corpo? } \\
\text { (T2) Você já sentiu dores em } \\
\text { outras partes do seu corpo? }\end{array}$ & $\mathrm{T} 2$ & $\begin{array}{l}\text { (R1) Have you had pain in } \\
\text { other parts of your body? } \\
\text { (R2) Have you had pain in } \\
\text { other parts of your body? }\end{array}$ & $\begin{array}{l}\text { Você teve dores } \\
\text { em outras partes } \\
\text { do seu corpo? }\end{array}$ \\
\hline 13 & $\begin{array}{l}\text { Have you had } \\
\text { allergic reactions? }\end{array}$ & $\begin{array}{l}\text { (T1) Você já teve reações } \\
\text { alérgicas? } \\
\text { (T2) Você já teve reações } \\
\text { alérgicas? }\end{array}$ & $\mathrm{T} 1=\mathrm{T} 2$ & $\begin{array}{l}\text { (R1) Have you had allergic } \\
\text { reactions? } \\
\text { (R2) Have you had allergic } \\
\text { reactions? }\end{array}$ & $\begin{array}{l}\text { Você teve reações } \\
\text { alérgicas? }\end{array}$ \\
\hline 14 & $\begin{array}{l}\text { Have you had } \\
\text { burning or sore } \\
\text { eyes? }\end{array}$ & $\begin{array}{l}\text { (T1) Teve ardência ou } \\
\text { irritação nos olhos? } \\
\text { (T2) Você já sentiu ardência } \\
\text { ou irritaçãa nos olhos? }\end{array}$ & $\mathrm{T} 2$ & $\begin{array}{l}\text { (R1) Have you had burning or } \\
\text { sore eyes? } \\
\text { (R2) Have you had burning } \\
\text { eyes? }\end{array}$ & $\begin{array}{l}\text { Você teve } \\
\text { ardência ou } \\
\text { irritação nos } \\
\text { olhos? }\end{array}$ \\
\hline
\end{tabular}


Translation and cultural adaptation of the European Organisation for Research and Treatment of Cancer Quality of Life Question-

Table 1. Continued...

\begin{tabular}{|c|c|c|c|c|c|}
\hline$\#$ & $\begin{array}{l}\text { Original English- } \\
\text { language version }\end{array}$ & Translation & $\begin{array}{l}\text { Selected } \\
\text { translation }\end{array}$ & Back-translation & $\begin{array}{c}\text { Final Brazilian } \\
\text { Portuguese- } \\
\text { language version }\end{array}$ \\
\hline 15 & $\begin{array}{l}\text { Have you been } \\
\text { dizzy? }\end{array}$ & $\begin{array}{l}\text { (T1) Você ficou tonto? } \\
\text { (T2) Você já se sentiu tonto? }\end{array}$ & $\mathrm{T} 2$ & $\begin{array}{l}\text { (R1) Have you been dizzy? } \\
\text { (R2) Have you felt dizzy? }\end{array}$ & $\begin{array}{l}\text { Você teve } \\
\text { tonturas? }\end{array}$ \\
\hline 16 & $\begin{array}{l}\text { Have you had } \\
\text { splitting fingernails } \\
\text { or toenails? }\end{array}$ & $\begin{array}{l}\text { (T1) Você já teve rachaduras } \\
\text { nas unhas das mãos ou pés? } \\
\text { (T2) Você já teve as unhas } \\
\text { das mãos ou pés quebradas? }\end{array}$ & $\mathrm{T} 2$ & $\begin{array}{l}\text { (R1) Have you had splitting } \\
\text { fingernails or toenails? } \\
\text { (R2) Have you had cracking } \\
\text { fingernails or toenails? }\end{array}$ & $\begin{array}{l}\text { Você teve as } \\
\text { unhas das mãos ou } \\
\text { pés quebradas? }\end{array}$ \\
\hline 17 & $\begin{array}{l}\text { Have you had skin } \\
\text { problems (e.g. } \\
\text { itchy, dry)? }\end{array}$ & $\begin{array}{l}\text { (T1) Você teve problemas } \\
\text { de pele (ex.: coceira, pele } \\
\text { seca)? } \\
\text { (T2) Você já teve problemas } \\
\text { na pele (ex: coceira, pele } \\
\text { seca)? }\end{array}$ & $\mathrm{T} 2$ & $\begin{array}{l}\text { (R1) Have you had skin } \\
\text { problems (e.g. itchy, dry)? } \\
\text { (R2) Have you had skin } \\
\text { problems (e.g. itchy, dry)? }\end{array}$ & $\begin{array}{l}\text { Você teve } \\
\text { problemas na pele } \\
\text { (ex.: coceira, pele } \\
\text { seca)? }\end{array}$ \\
\hline 18 & $\begin{array}{l}\text { Have you had } \\
\text { problems speaking? }\end{array}$ & $\begin{array}{l}\text { (T1) Sentiu dificuldades para } \\
\text { falar? } \\
\text { (T2) Você já sentiu } \\
\text { dificuldades para falar? }\end{array}$ & $\mathrm{T} 2$ & $\begin{array}{l}\text { (R1) Have you had problems } \\
\text { speaking? } \\
\text { (R2) Have you had problems } \\
\text { speaking? }\end{array}$ & $\begin{array}{l}\text { Você teve } \\
\text { problemas para } \\
\text { falar? }\end{array}$ \\
\hline 19 & $\begin{array}{l}\text { Have you been } \\
\text { afraid of tumor } \\
\text { progression? }\end{array}$ & $\begin{array}{l}\text { (T1) Tem medo da progressão } \\
\text { tumoral? } \\
\text { (T2) Você tem medo da } \\
\text { progressão do tumor? }\end{array}$ & $\mathrm{T} 2$ & $\begin{array}{l}\text { (R1) Have you been afraid of } \\
\text { tumor progression? } \\
\text { (R2) Have you been afraid of } \\
\text { tumor progression? }\end{array}$ & $\begin{array}{l}\text { Você teve medo } \\
\text { da progressão do } \\
\text { tumor? }\end{array}$ \\
\hline 20 & $\begin{array}{l}\text { Have you had } \\
\text { thin or lifeless } \\
\text { hair as a result of } \\
\text { your disease or } \\
\text { treatment? }\end{array}$ & $\begin{array}{l}\text { ( } \mathrm{T} 1 \text { ) Você teve um } \\
\text { afinamento do cabelo ou o } \\
\text { mesmo ficou sem vida como } \\
\text { resultado do tratamento ou } \\
\text { da sua doença? } \\
\text { (T2) Você teve um cabelo } \\
\text { mais fino ou sem vida como } \\
\text { resultado da doença ou do } \\
\text { tratamento? }\end{array}$ & $\mathrm{T} 1$ & $\begin{array}{l}\text { (R1) Have you had thin or } \\
\text { lifeless hair as a result of } \\
\text { your disease or treatment? } \\
\text { (R2) Have you had thin or } \\
\text { lifeless hair as a result of } \\
\text { your illness or treatment? }\end{array}$ & $\begin{array}{l}\text { Você teve um } \\
\text { afinamento do } \\
\text { cabelo ou o } \\
\text { mesmo ficou } \\
\text { sem vida como } \\
\text { resultado de } \\
\text { sua doença ou } \\
\text { tratamento? }\end{array}$ \\
\hline 21 & $\begin{array}{l}\text { Have you worried } \\
\text { about your health } \\
\text { in the future? }\end{array}$ & $\begin{array}{l}\text { (T1) Você já se preocupa com } \\
\text { sua saúde no futuro? } \\
\text { (T2) Você tem se preocupado } \\
\text { com sua saúde no futuro? }\end{array}$ & $\mathrm{T} 2$ & $\begin{array}{l}\text { (R1) Have you worried about } \\
\text { your health in the future? } \\
\text { (R2) Have you worried about } \\
\text { your health in the future? }\end{array}$ & $\begin{array}{l}\text { Você teve } \\
\text { preocupações } \\
\text { com sua saúde no } \\
\text { futuro? }\end{array}$ \\
\hline 22 & $\begin{array}{l}\text { Have you had dry } \\
\text { cough? }\end{array}$ & $\begin{array}{l}\text { (T1) Você teve tosse seca? } \\
\text { (T2) Você já teve tosse seca? }\end{array}$ & $\mathrm{T} 2$ & $\begin{array}{l}\text { (R1) Have you had dry cough? } \\
\text { (R2) Have you had dry cough? }\end{array}$ & $\begin{array}{l}\text { Você teve tosse } \\
\text { seca? }\end{array}$ \\
\hline 23 & $\begin{array}{l}\text { Have you } \\
\text { experienced } \\
\text { a decrease in } \\
\text { your physical } \\
\text { capabilities? }\end{array}$ & $\begin{array}{l}\text { (T1) Você sofreu uma } \\
\text { diminuição nas suas } \\
\text { capacidades físicas? } \\
\text { (T2) Você sentiu que sua } \\
\text { capacidade física tem } \\
\text { diminuído? }\end{array}$ & $\mathrm{T} 2$ & $\begin{array}{l}\text { (R1) Have you noticed a } \\
\text { decrease in your physical } \\
\text { capabilities? } \\
\text { (R2) Have you noticed a } \\
\text { decrease in your physical } \\
\text { capacities? }\end{array}$ & $\begin{array}{l}\text { Você teve a } \\
\text { sensação de que } \\
\text { sua capacidade } \\
\text { física diminuiu? }\end{array}$ \\
\hline 24 & $\begin{array}{l}\text { Has weight loss } \\
\text { been a problem for } \\
\text { you? }\end{array}$ & $\begin{array}{l}\text { (T1) A perda de peso foi um } \\
\text { problema para você? } \\
\text { (T2) A perda de peso tem sido } \\
\text { um problema para você? }\end{array}$ & $\mathrm{T} 2$ & $\begin{array}{l}\text { (R1) Has weight loss been a } \\
\text { problem for you? } \\
\text { (R2) Has weight loss been a } \\
\text { problem for you? }\end{array}$ & $\begin{array}{l}\text { A perda de peso } \\
\text { foi um problema } \\
\text { para você? }\end{array}$ \\
\hline 25 & $\begin{array}{l}\text { Have you had pain } \\
\text { in the area of } \\
\text { surgery? }\end{array}$ & $\begin{array}{l}\text { (T1) Você sofreu dor na área } \\
\text { de cirurgia? } \\
\text { (T2) Você já sentiu dores na } \\
\text { área da cirurgia? }\end{array}$ & $\mathrm{T} 2$ & $\begin{array}{l}\text { (R1) Have you had pain in the } \\
\text { area of surgery? } \\
\text { (R2) Have you had pain in the } \\
\text { area of surgery? }\end{array}$ & $\begin{array}{l}\text { Você teve dores } \\
\text { na área da } \\
\text { cirurgia? }\end{array}$ \\
\hline 26 & $\begin{array}{l}\text { Has the area of } \\
\text { your wound been } \\
\text { oversensitive? }\end{array}$ & $\begin{array}{l}\text { (T1) A área da sua lesão foi } \\
\text { muito sensível? } \\
\text { (T2) A área da sua ferida } \\
\text { ficou muito sensível? }\end{array}$ & $\mathrm{T} 2$ & $\begin{array}{l}\text { (R1) Has the area of your } \\
\text { wound been oversensitive? } \\
\text { (R2) Has the area of your } \\
\text { lesion been highly sensitive? }\end{array}$ & $\begin{array}{l}\text { A área da sua } \\
\text { ferida ficou muito } \\
\text { sensível? }\end{array}$ \\
\hline 27 & $\begin{array}{l}\text { Have you been } \\
\text { restricted in your } \\
\text { performance due } \\
\text { to the extent of } \\
\text { surgery? }\end{array}$ & $\begin{array}{l}\text { (T1) Você foi restringido em } \\
\text { seu desempenho devido à } \\
\text { extensão da cirurgia? } \\
\text { (T2) Você teve seu } \\
\text { desempenho limitado pela } \\
\text { extensão da cirurgia? }\end{array}$ & $\mathrm{T} 2$ & $\begin{array}{l}\text { (R1) Have you been restricted } \\
\text { in your performance due to } \\
\text { the extent of surgery? } \\
\text { (R2) Have you had your } \\
\text { performance limited due to } \\
\text { the extent of surgery? }\end{array}$ & $\begin{array}{l}\text { Você teve } \\
\text { restrição do seu } \\
\text { desempenho } \\
\text { devido à extensão } \\
\text { da cirurgia? }\end{array}$ \\
\hline
\end{tabular}


Table 1. Continued...

\begin{tabular}{|c|c|c|c|c|c|}
\hline$\#$ & $\begin{array}{l}\text { Original English- } \\
\text { language version }\end{array}$ & Translation & $\begin{array}{l}\text { Selected } \\
\text { translation }\end{array}$ & Back-translation & $\begin{array}{c}\text { Final Brazilian } \\
\text { Portuguese- } \\
\text { language version }\end{array}$ \\
\hline 28 & $\begin{array}{l}\text { Have you had } \\
\text { any difficulty } \\
\text { using your arm or } \\
\text { shoulder on the } \\
\text { side of the chest } \\
\text { operation? }\end{array}$ & $\begin{array}{l}\text { (T1) Você teve alguma } \\
\text { dificuldade em usar seu } \\
\text { braço ou ombro no lado da } \\
\text { operação do peito? } \\
\text { (T2) Você teve alguma } \\
\text { dificuldade de movimentar } \\
\text { o braço ou ombro no lado da } \\
\text { cirurgia? }\end{array}$ & $\mathrm{T} 2$ & $\begin{array}{l}\text { (R1) Have you had any } \\
\text { difficulty using your arm or } \\
\text { shoulder on the side of the } \\
\text { chest operation? } \\
\text { (R2) Have you had any } \\
\text { difficulty moving your arm or } \\
\text { shoulder on the side of the } \\
\text { chest operation? }\end{array}$ & $\begin{array}{l}\text { Você teve alguma } \\
\text { dificuldade de } \\
\text { usar o braço ou } \\
\text { ombro no lado da } \\
\text { cirurgia? }\end{array}$ \\
\hline 29 & $\begin{array}{l}\text { Has your scar } \\
\text { pain interfered } \\
\text { with your daily } \\
\text { activities? }\end{array}$ & $\begin{array}{l}\text { (T1) Sua dor de cicatriz } \\
\text { interferiu com suas } \\
\text { atividades diárias? } \\
\text { (T2) A dor da sua cicatriz } \\
\text { tem interferido em suas } \\
\text { atividades diárias? }\end{array}$ & $\mathrm{T} 2$ & $\begin{array}{l}\text { (R1) Has your scar pain } \\
\text { interfered with your daily } \\
\text { activities? } \\
\text { (R2) Has your scar pain } \\
\text { interfered with your daily } \\
\text { activities? }\end{array}$ & $\begin{array}{l}\text { A dor da sua } \\
\text { cicatriz interferiu } \\
\text { em suas atividades } \\
\text { diárias? }\end{array}$ \\
\hline & $\begin{array}{l}\text { Were there any } \\
\text { symptoms or } \\
\text { problems that were } \\
\text { not covered by } \\
\text { the questionnaire, } \\
\text { but were relevant } \\
\text { for you in the past } \\
\text { week? }\end{array}$ & $\begin{array}{l}\text { (T1) Ocorreram quaisquer } \\
\text { sintomas ou problemas que } \\
\text { não foram abordados pelo } \\
\text { questionário, mas foram } \\
\text { relevantes para você na } \\
\text { semana passada? } \\
\text { (T2) Aconteceram qualquer } \\
\text { outro sintoma ou problema } \\
\text { que não foram abordados } \\
\text { neste questionário, mas que } \\
\text { foram importantes para você, } \\
\text { na semana passada? }\end{array}$ & $\mathrm{T} 1$ & $\begin{array}{l}\text { (R1) Were there any } \\
\text { symptoms or problems that } \\
\text { were not covered by the } \\
\text { questionnaire, but were } \\
\text { relevant for you in the past } \\
\text { week? } \\
\text { (R2) Were there any } \\
\text { symptoms or problems that } \\
\text { were not covered by the } \\
\text { questionnaire, but were } \\
\text { relevant for you in the last } \\
\text { week? }\end{array}$ & $\begin{array}{l}\text { Ocorreram } \\
\text { quaisquer } \\
\text { sintomas ou } \\
\text { problemas } \\
\text { que não foram } \\
\text { abordados pelo } \\
\text { questionário, mas } \\
\text { foram relevantes } \\
\text { para você na } \\
\text { última semana? }\end{array}$ \\
\hline
\end{tabular}

All patients completed the questionnaire in less than 25 minutes, in a designated area within the hospital. Once pilot testing was completed, the comments from patients were analyzed. No difficulties in answering the questions were reported, and none of the items were found to be confusing, upsetting, or offensive, or to contain difficult vocabulary. Therefore, no changes were made to the final version approved by the EORTC translation unit. The Portuguese-language version of the QLQ-LC29 was approved. The steps are described in Table 1.

The understandability of the instrument was good, with a mean of 5.0 points (maximum value of 5.0 ), and most questions were fully understood (Table 2). The internal consistency of the scale was calculated with Cronbach's alpha coefficient, and an alpha value of 0.94 was found.

The full version of the instrument cannot be published in the present study for copyright reasons. The final version of the instrument can be purchased by consulting the EORTC.

\section{DISCUSSION}

This study presents data regarding the translation of the EORTC QLQ-LC29 to Portuguese in Brazil and regarding the cultural adaptation and pilot testing of this Portuguese-language version. This is the first such version, and was authorized and audited by the EORTC, which oversaw all the steps in creating this Portuguese-language version.

The importance of making the EORTC QLQ-LC29 available to scientists and clinicians in the field of oncology is immeasurable, since lung cancer is the most common cancer and is the one with the highest mortality rates and the lowest 5-year survival rates, ${ }^{(3-5)}$ and therefore it is important that patient QoL be a variable taken into account. ${ }^{(6,8)}$ Through the use of a disease-specific tool such as the EORTC QLQ-LC29, it is possible not only to predict patient prognosis or patient morbidity and mortality, but also to inform decisions regarding treatment, especially because the questionnaire provides information about patient clinical status in various domains. ${ }^{(6,11,12,15)}$

The measurement properties of the original Englishlanguage version of the EORTC QLQ-LC29 were evaluated and verified at the time of its creation in a multicenter study. ${ }^{(11)}$ Because the EORTC QLQ-LC29 is a recent module, this is its first translated version, and therefore data are lacking for a comparison between our results and those of other studies. It is expected that, as soon as the original English-language version of the EORTC QLQ-LC29 is widely disseminated, various researchers from different countries and speaking different languages will translate and adapt this module in order to make this disease-specific tool available for assessing QoL in patients with lung cancer. ${ }^{(11)}$

For each new translation, a series of cultural changes and adaptations are made in order to develop a version specific to a given population and its characteristics. ${ }^{(16,17)}$ This specificity justifies the need for translations and cultural adaptations, considering that a given topic can elicit different answers and different effects because of cultural differences. ${ }^{(10)}$ 
Translation and cultural adaptation of the European Organisation for Research and Treatment of Cancer Quality of Life Questionnaire-Lung Cancer Module for quality of life assessment in patients with lung cancer in Brazil

Table 2. Assessment of the understandability of the Brazilian Portuguese-language version of the Quality of Life Questionnaire-Lung Cancer 29.

\begin{tabular}{cccc} 
Question & Understandability, mean (SD) & Question & Understandability, mean (SD) \\
1 & $5.0(0.0)$ & 16 & $5.0(0.0)$ \\
2 & $5.0(0.0)$ & 17 & $5.0(0.0)$ \\
3 & $5.0(0.0)$ & 18 & $5.0(0.0)$ \\
4 & $5.0(0.0)$ & 19 & $5.0(0.0)$ \\
5 & $5.0(0.0)$ & 20 & $5.0(0.0)$ \\
6 & $5.0(0.0)$ & 21 & $4.8(0.4)$ \\
7 & $5.0(0.0)$ & 22 & $5.0(0.0)$ \\
8 & $5.0(0.0)$ & 23 & $4.9(0.4)$ \\
9 & $5.0(0.0)$ & 24 & $5.0(0.0)$ \\
10 & $5.0(0.0)$ & 25 & $5.0(0.0)$ \\
11 & $5.0(0.0)$ & 26 & $5.0(0.0)$ \\
12 & $5.0(0.0)$ & 27 & $4.5(0.5)$ \\
13 & $4.7(0.5)$ & 28 & $5.0(0.0)$ \\
14 & $5.0(0.0)$ & 29 & $5.0(0.0)$ \\
15 & $5.0(0.0)$ & & \\
\hline
\end{tabular}

We acknowledge that our study may have some limitations, such as the size of the pilot-testing sample, which was intentionally selected; however, sampling was carried out in accordance with the EORTC recommendations. ${ }^{(13,18)}$ Despite being small, the sample was sufficient to validate the translated version according to the EORTC recommendations. Data collection with a larger sample would make it possible to perform more complex analyses, including analysis of measurement properties, in accordance with parameters used internationally. ${ }^{(18)}$

After completion of all the steps described in the present study and analysis of the results, our data suggest that the present Portuguese-language version of the EORTC QLQ-LC29 is suitable for use by scientists and clinicians in Brazil.

\section{REFERENCES}

1. Ridge CA, McErlean AM, Ginsberg MS. Epidemiology of Lung Cancer. Semin Intervent Radiol. 2013;30(2):93-8. https://doi. org/10.1055/s-0033-1342949

2. Bray F, Ferlay J, Soerjomataram I, Siegel RL, Torre LA, Jema A. Global cancer statistics 2018: Global cancer statistics 2018 GLOBOCAN estimates of incidence and mortality worldwide for 36 cancers in 185 countries. CA Cancer J Clin. 2018;68(6):394-424. https://doi.org/10.3322/caac.21492

3. Instituto Nacional de Câncer. Estimativa 2018: incidência de cânce no Brasil. Rio de Janeiro: Instituto Nacional de Câncer; 2018.

4. Kawaguchi T, Ando M, Ito N, Isa SI, Tamiya A, Shimizu S, et al. Rationale and design of the Japan molecular epidemiology for lung cancer study. Clin Lung Cancer. 2013;14(5):596-600. https://doi. org/10.1016/j.cllc.2013.03.001

5. Syrjänen K. Detection of human papillomavirus in lung cancer: systematic review and meta-analysis. Anticancer Res. 2012;32(8):3235-50.

6. Bottomley A. The cancer patient and quality of life. Oncologist 2002;7(2):120-5. https://doi.org/10.1634/theoncologist.7-2-120

7. Fallowfield L. Quality of life: a new perspective for cancer patients Nat Rev Cancer. 2002;2(11):873-9. https://doi.org/10.1038/nrc930

8. Bottomley A, Aaronson NK; European Organisation for Research and Treatment of Cancer. International perspective on healthrelated quality-of-life research in cancer clinical trials: the European Organisation for Research and Treatment of Cancer experience. J Clin Oncol. 2007;25(32):5082-6. https://doi.org/10.1200/ JCO.2007.11.3183

9. Aaronson NK, Ahmedzai S, Bergman B, Bullinger M, Cull A, Duez $\mathrm{NJ}$, et al. The European Organization for Research and Treatment of Cancer QLQ-C30: a quality-of-life instrument for use in international clinical trials in oncology. J Natl Cancer Inst. 1993;85(5):365-76. https://doi.org/10.1093/jnci/85.5.365

10. Relvas-Silva M, Silva RA, Dinis-Ribeiro M. Portuguese Version of the EORTC QLQ-OES18 and QLQ-OG25 for Health-Related Quality

of Life Assessment. Acta Med Port. 2017;30(1):47-52. https://doi. org/10.20344/amp.7499

11. Koller M. Update of the EORTC questionnaire for assessing quality of life in patients with lung cancer: Introducing the new EORTC QLQLC29. J Clin Oncol. 2016;34(15_suppl):e18096.

12. Koller M, Hjermstad MJ, Tomaszewski KA, Tomaszewska IM Hornslien K, Harle A, et al. An international study to revise the EORTC questionnaire for assessing quality of life in lung cancer patients. Ann Oncol. 2017;28(11):2874-2881. https://doi.org/10.1093/ annonc/mdx453

13. Kuliś D, Bottomley A, Velikova G, Greimel E, Koller M. EORTC Quality of Life Group Translation Procedure. 4th ed. Brussels: EORTC; 2017.

14. Conti MA, Tavares H, Jardim AP, Hearst N, Cordás TA, Abreu CN Evaluation of semantic equivalence and internal consistency of a Portuguese version of the Internet Addiction Test (IAT). Rev Psiquatr Clin (Sao Paulo). 2012;39(3):106-110. https://doi.org/10.1590/s0101 60832012000300007

15. Pennathur A, Gibson MK, Jobe BA, Luketich JD. Oesophagea carcinoma. Lancet. 2013;381(9864):400-12. https://doi.org/10.1016/ S0140-6736(12)60643-6

16. Scott NW, Fayers PM, Bottomley A, Aaronson NK, de Graeff $A$, Groenvold $M$, et al. Comparing translations of the EORTC QLQ-C30 using differential item functioning analyses. Qual Life Res. 2006;15(6):1103-15; discussion 1117-20. https://doi.org/10.1007/ s11136-006-0040-x

17. Scott NW, Fayers PM, Aaronson NK, Bottomley A, de Graeff A, Groenvold $M$, et al. The relationship between overall quality of life and its subdimensions was influenced by culture: analysis of an international database. J Clin Epidemiol. 2008;61(8):788-95. https:// doi.org/10.1016/j.jclinepi.2007.08.015

18. Terwee CB, Bot SD, de Boer MR, van der Windt DA, Knol DL, Dekker $J$, et al. Quality criteria were proposed for measurement properties of health status questionnaires. J Clin Epidemiol. 2007;60(1):34-42. https://doi.org/10.1016/j.jclinepi.2006.03.012 